\title{
The effect of optical blur on visual-geometric illusions
}

\author{
STANLEY COREN and LAWRENCE M. WARD \\ University of British Columbia, Vancouver, British Columbia, Canada \\ and \\ CLARE PORAC and ROBERT FRASER \\ University of Victoria, Victoria, British Columbia, Canada
}

\begin{abstract}
Blur, due to optical aberrations in the eye, has been implicated in visual illusions for converging line arrays. Using 26 illusion variants, 2.5 diopters of optical blur was optically induced, resulting in increased illusory effects for the Poggendorff and several Mueller-Lyer variants, but not for the other configurations. Some limitations on conditions drawn from induced blur are discussed.
\end{abstract}

Over the past several years, it has become apparent that visual-geometric illusions are caused by a number of different interacting mechanisms, operating at different levels in the visual system (Coren \& Girgus, 1972; Coren, Girgus, Ehrlichman, \& Hakstian, 1976; Girgus, Coren, Durant, \& Porac, 1975). We may roughly divide these mechanisms into two classes. The first we will call structural, encompassing optical and neural mechanisms which can lead to illusory distortions. The second we will call strategy, which will include cognitivejudgmental effects that introduce bias in the processing of information at higher levels.

Some structural mechanisms capable of inducing illusory distortions operate quite early in the visual system. In fact, at the first interface between the stimulus and the observer, the dioptric system of the eye begins to alter the stimulus pattern. Chiang (1968) has shown that blurring of the retinal image caused by spherical and chromatic aberrations of the crystalline lens, or defractions of light at the pupillary aperture, could cause illusory effects in intersecting line patterns such as the Mueller-Lyer (Figure 1L) or the Poggendorff (Figure 1A) illusions. If the locus of a contour is taken to be the maximum of the local distribution of light in a pattern, then the effect of this blur would be to shift the vertex of an acute angle into the body of the angle and to render acute angles slightly more obtuse.

Coren (1969) directly tested the optical contribution to the Poggendorff illusion by reducing the amount of blur in the retinal image through the use of artificial pupils and narrow-band chromatic filters. He reported that this treatment reduced the illusion. He estimated that $22 \%$ of the total magnitude of the Poggendorff may be caused by such blur effects. More recently,

This research was supported by grants from the National Research Council and Medical Research Council of Canada.
Ward and Coren (1976) reversed this procedure. They introduced blur into the Mueller-Lyer illusion and showed that the magnitude of the distortion increased. Although these results seem to suggest that blur can be a factor in illusion formation, there are some hints that the expected blur effects do not take place in all intersecting line configurations. For example, Coren (1970a) modified the Mueller-Lyer and the Poggendorff configurations so that they contained dots only at line ends and vertices. With no converging and intersecting line elements, no blur interactions can occur, hence one would expect a reduction in illusion magnitude for such dotted displays. Coren found such a reduction but, when a similar manipulation was performed on the Wundt-Hering illusion (Coren, 1970b), no reduction in illusory distortion occurred. This implies that blur effects contribute to illusion magnitude in only a limited set of arrays and that the simple existence of converging line elements is not sufficient to guarantee that blur is a factor in illusion formation. Since direct manipulation of the amount of optical blur has been attempted on only a limited set of illusion configurations, the following experiment was conducted to see how induced image blur affects illusion strength in a variety of different figures.

\section{METHOD}

A set of 26 of the most commonly encountered visualgeometric illusions arrays were selected. These included the Poggendorff (1A), Wundt (1B), Zoellner (1C), Jastrow (1D), and the over- and underestimated segments of the Ebbinghaus (1E), Delboeuf (1F), Ponzo (1G), Baldwin (1H), horizontalvertical (1I), Sander parallelogram (1J), and the Oppel-Kundt (1K). The set also included the over- and underestimated segments of four variants of the Mueller-Lyer illusion, including the standard form (1L), Coren's dot form (1M), Piaget's line form (1N), and the box wing form (10).

Stimuli were prepared as transparencies which could be shown using an overhead projector. The average extent of the 


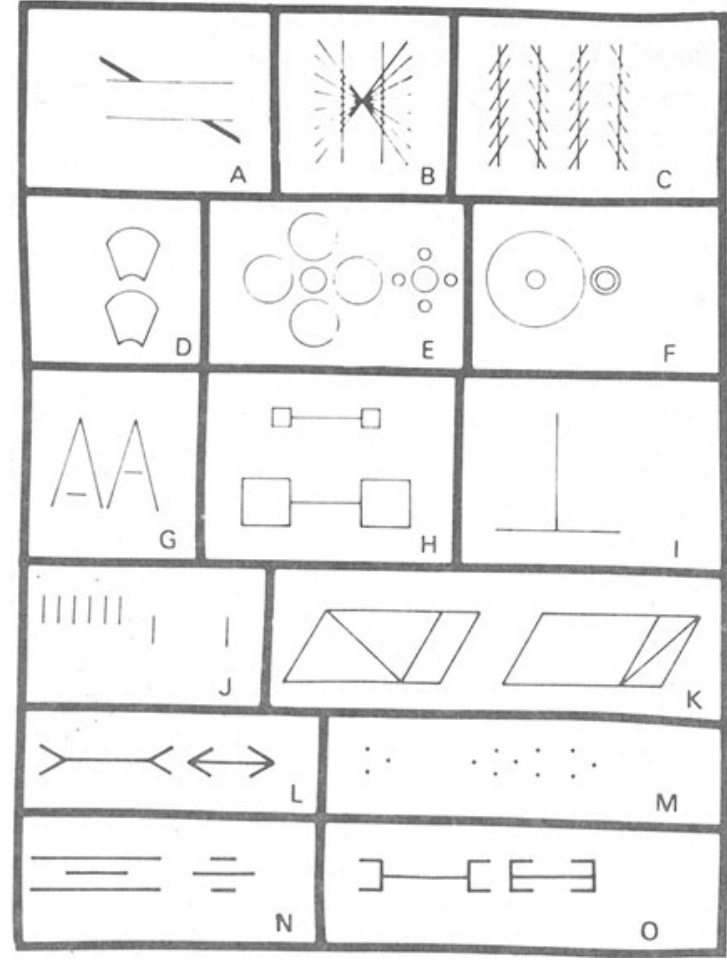

Figure 1. The illusion set tested: (A) Poggendorff, (B) Wund : (C) Zoellner, (D) Jastrow, (E) Ebbinghaus, (F) Delboeuf, (G) Ponzo, (H) Baldwin, (I) horizontal-vertical, (J) Oppel-Kundt, (K) Sander parallelogram, (L) standard Mueller-Lyer, (M) Coren's dot ML, (N) Piaget's line ML, (O) box wing ML.

projected stimuli was 12-deg visual angle. Coren et al. (1976) have shown that all of the visual illusions used in this set can be measured using judgments of the linear extent of certain critical aspects of the array. An adjustable line length was projected upon the upper left segment of the screen. The length of this line was adjusted by the subject until it appeared to match the critical extents as defined in Coren et al. (1976). All subjects were shown the various illusion configurations in mixed order. Each stimulus was presented in sharp focus or blurred by 2.5 diopters. Although the stimulus appeared in either blurred or unblurred presentations, the adjustable line was always in clear focus. For those stimuli containing an over- and an underestimated portion, each segment of the illusion was separately presented and judged.

Twenty-eight paid observers served as subjects. Observers were limited to those individuals who had tested visual acuity of $20 / 25$ or better in order to control for undetected refractive errors which might interact with the blur manipulation (Coren \& Porac, 1975).

\section{RESULTS AND DISCUSSION}

The differences between the judgments on the blurred and nonblurred stimuli were computed for each configuration. These are shown in Table 1. Where figures contain an over- and underestimated segment, the estimations are indicated by $\mathrm{O}$ or $\mathrm{U}$, respectively. Positive values indicate a greater illusory effect under blurred conditions than under nonblurred conditions, and negative values indicate a reduction of illusion under blur presentation. All starred values in the table represent differences that are statistically significant at $\mathrm{p}<.05$ or better.

As can be seen from the table, blur did not significantly affect the judgment of length of a control line. Significant increases in illusion magnitude were obtained under blurred conditions for the Poggendorff and the standard form of the Mueller-Lyer illusion. This replicates earlier reports (Coren, 1969; Ward \& Coren, 1976). According to Chiang's (1968) formulation of the involvement of blur in illusion formation, both the Wundt-Hering and the Zoellner configuration should also increase in magnitude under blur, as both arrays involve many intersecting line elements. However, neither of the configurations shows any increase in illusory effect. This result is consistent with Coren's (1970b) failure to change illusion magnitude in the Wundt-Hering by eliminating the converging line elements. The Sander parallelogram shows a puzzling decrease in illusion magnitude for its usually overestimated segment.

When we look at the configurations which do not have converging and intersecting line elements, we find that, as predicted, the introduction of optical blur does not alter the magnitude of the distortion for most of

Table 1

The Effect of Blur on Illusion Magnitude

\begin{tabular}{lc} 
& $\begin{array}{c}\text { Illusion Change } \\
\text { (in millimeters) }\end{array}$ \\
\hline Poggendorff & $11.4^{*}$ \\
Wundt & .9 \\
Zoellner & 1.1 \\
Jastrow & .7 \\
Ebbinghaus O & 2.9 \\
Ebbinghaus U & .0 \\
Delboeuf O & .2 \\
Delboeuf U & 3.0 \\
Ponzo O & .0 \\
Ponzo U & .3 \\
Baldwin O & $12.1^{*}$ \\
Baldwin U & 4.2 \\
Horizontal-Vertical O & .2 \\
Horizontal-Vertical U & .1 \\
Sander Parallelogram O & $-13.7^{*}$ \\
Sander Parallelogram U & 5.4 \\
Oppel-Kundt O & 1.1 \\
Oppel-Kundt U & .1 \\
Standard Mueller-Lyer O & $15.2^{*}$ \\
Standard Mueller-Lyer U & 8.3 \\
Coren's Dot Mueller-Lyer O & -.4 \\
Coren's Dot Mueller-Lyer U & -3.2 \\
Piaget's Line Mueller-Lyer O & $7.5^{*}$ \\
Piaget's Line Mueller-Lyer U & 7.2 \\
Box Wing Mueller-Lyer O & $5.6^{*}$ \\
Box Wing Mueller-Lyer O & $8.2^{*}$ \\
Control Line & .1 \\
\hline
\end{tabular}

*Indicates statistically significant with $p<.05$. Indicates illusion reduction with blur, $O$ indicates overestimated segment, $U$ indicates underestimated segment. 
these arrays. All of the exceptions to this generalization, however, are Mueller-Lyer variants (one may consider the overestimated version of the Baldwin illusion, in terms of configuration, as simply the box wing MuellerLyer with the ends closed).

Though one could have predicted, from geometrical considerations, the illusion increase with blur for the standard Mueller-Lyer, the increased illusion for both segments of the box wing variant, and the overestimated Piaget variant are not predictable from considerations of overlapping light distributions in the retinal image. These results seem to suggest that some judgmental strategy mechanism has been evoked by the presence of the degraded stimuli. The most likely candidates are based upon global impression theories, such as Pressey's $(1970,1971)$ assimilation theory or Erlebacher and Sekuler's (1969) confusion theory. In these theories the test elements take on some of the qualities (in terms of extent, locus, or direction) of the ancillary lines. If elements of the inducing lines are merged or confused with aspects of the test lines as part of the illusionformation process, then blurring the array will make the regions where the test extent ends and inducer begins less clear. This could result in an increased illusion for figures in which this mechanism is operating.

The results of this direct optical manipulation technique show that blur does not affect a large number of illusion figures which do not contain intersecting line elements. Also, expected increases in the Wundt and Zoellner arrays were not found. The fact that induced blur also affects some illusions which should not be subject to the effects of optical smearing suggests that strategy, as well as structural, mechanisms may also be altered using this manipulation. Direct blurring techniques may well change too much by altering the appearance of the array, as well as the light distribution, in the final retinal image. Thus, data showing illusion increases resulting from direct optical blurring of the image may be taken as only weak support for structural factors in illusion formation. More positively, however, the present results do set tentative boundary conditions on the presence of optical blur effects in visual-geometric illusions. The absence of blur effects on some figures seems to rule out optical factors as a mechanism for these configurations, while the appearance of blur effects is only suggestive evidence for optical components in illusion formation.

\section{REFERENCES}

ChIANG. C. A new theory to explain geometrical illusions produced by crossing lines. Perception \& Psychophysics, 1968, 3. 174-176.

COREN. S. The influence of optical aberrations on the magnitude of the Poggendorff illusion. Perception \& Psychophysics, 1969, 6, 185-186.

Coren, $\dot{S}$. Lateral inhibition and geometric illusions. Quarterly' Journal of Experimental Psychology, 1970, 22. 274-278. (a)

Coren, S. Lateral inhibition and the Wundt-Hering illusion. Psichonomic Science, 1970, 18, 341. (b)

Coren, S., \& Girgus, J. S. Illusion decrement in intersecting line figures. Psychonomic Science, 1972, 26, 108-110.

Coren, S., Girgus, J. S., Ehrlichman, H., \& Hakstian, A. R. An empirical taxonomy of visual illusions. Perception \& Psychophysics, 1976, 20, 129-137.

Coren, S.. \& Porac, C. The myth of the normal eye: A methodological note. Bulletin of the Psychonomic Society. 1975, 5. 469-470.

Erlebacher, A., \& Sekuler, R. Explanation of the MuellerLyer illusion: Confusion theory examined. Journal of Experimental Psychology, 1969, 80. 462-467.

Girgus, J. S.. Coren, S., Durant, M.. \& Porac, C. The assessment of components involved in illusion formation using a long-term decrement procedure. Perception \& Psychophysics, 1975, 18, 144-148.

Pressey, A. W. The assimilation theory applied to a modification of the Mueller-Lyer illusion. Perception \& Psychophysics, 1970, 8, 411-412.

PRESSEY, A. W. An extension of assimilation theory to illusions of size, area and direction. Perception \& Psychophysics. 1971, 9. 172-176.

WARD, L. M., \& COREN, S. The effect of optically induced blur on the magnitude of the Mueller-Lyer illusion. Bulletin of the Psychonomic Society, 1976, 7, 483-484.

(Received for publication February 24, 1978.) 